\title{
Abstract: Deep Learning-based Quantification of Pulmonary Hemosiderophages in Cytology Slides
}

\author{
Christian Marzahl ${ }^{1,2}$, Marc Aubreville ${ }^{1}$, Christof A. Bertram ${ }^{3}$, Jason Stayt ${ }^{4}$, \\ Anne Katherine Jasensky ${ }^{5}$, Florian Bartenschlager ${ }^{3}$, Marco Fragoso ${ }^{3}$, \\ Ann K. Barton ${ }^{6}$, Svenja Elsemann ${ }^{7}$, Samir Jabari ${ }^{8}$, Jens Krauth ${ }^{2}$, \\ Prathmesh Madhu ${ }^{1}$, Jörn Voigt ${ }^{2}$, Jenny Hill ${ }^{4}$, Robert Klopfleisch ${ }^{3}$, \\ Andreas Maier ${ }^{1}$ \\ ${ }^{1}$ Pattern Recognition Lab, Department of Computer Science, \\ Friedrich-Alexander-Universität Erlangen-Nürnberg (FAU), Germany \\ ${ }^{2}$ R \& D Projects, EUROIMMUN Medizinische Labordiagnostika AG \\ ${ }^{3}$ Institute of Veterinary Pathology, Freie Universität Berlin, Germany \\ ${ }^{4}$ VetPath Laboratory Services, Ascot,Western Australia \\ ${ }^{5}$ Laboklin GmbH und Co. KG, Bad Kissingen, Germany \\ ${ }^{6}$ Equine Clinic, Freie Universität Berlin, Berlin, Germany \\ ${ }^{7}$ Department of Neurosurgery, Universitätsklinikum Erlangen, Erlangen, Germany \\ ${ }^{8}$ Institute of Neuropathology, Universitätsklinikum Erlangen, Erlangen, Germany \\ c.marzahl@euroimmun.de
}

Exercise-induced pulmonary hemorrhage (EIPH) is a common condition in sport horses with negative impact on performance. Cytology of bronchoalveolar lavage fluid by use of a scoring system is considered the most sensitive diagnostic method. Manual grading of macrophages, depending on the degree of cytoplasmic hemosiderin content, on whole slide images (WSI) is however monotonous and time-consuming. We evaluated state-of-the-art deep learning-based methods for macrophage classification and compared them against the performance of nine cytology experts. Additionally, we evaluated object detection methods on a novel data set of 17 completely annotated cytology WSI containing 78,047 hemosiderophages [1]. Our deep learning-based approach reached a concordance of 0.85 , partially exceeding human expert concordance $(0.68$ to 0.86 , mean of 0.73 , SD of 0.04 ). Our object detection approach has a mean average precision of 0.66 over the five classes from the whole slide gigapixel images. To mitigate the high inter- and intra-rater variability, we propose our automated object detection pipeline, enabling accurate and reproducible EIPH scoring in WSI.

\section{References}

1. Marzahl C, Aubreville M, Bertram CA, et al. Deep learning-based quantification of pulmonary hemosiderophages in cytology slides. Sci Rep. 2020;10(1):1-10. 